\title{
Ökonomie und Gerinnungsmanagement
}

\author{
Thomas Ehrlich \\ Medizinisches System, Klinik Hirslanden, Zürich, Schweiz
}

Schlïsselwörter

Gerinnungsmanagement · G-DRG-System .

Point-of-Care-Diagnostik

\section{Zusammenfassung}

Hintergrund: Bei erworbenen oder chronisch bestehenden Störungen der Gerinnung ist in der perioperativen Phase eine gezielte Therapie von herausragender Bedeutung. Der kostenintensive Einsatz von Blut- und Gerinnungspräparaten und die Beeinflussung des Patientenoutcomes werden hinsichtlich des G-DRG(German Diagnosis Related Groups)-Systems und der Anwendung von Point-of-Care-Geräten bewertet. Methode: Anhand einer Literaturübersicht und eigener Daten wird der aktuelle Sachstand analysiert und diskutiert. Ergebnisse: Die perioperative Behandlung von Störungen der Gerinnung ist komplex. Neben der klassischen Labordiagnostik unterstützen und beschleunigen Point-of-Care-Geräte die Therapieentscheidungen. Die Anwendung eines Algorithmus-basierten Applikationsregimes von Blut- und Gerinnungspräparaten optimiert die Verbrauchsmengen und verbessert das Patientenoutcome. Demgegenüber können durch das G-DRG-System bedingte finanzielle Effekte aufgrund von kosten- und erlösseitigen Änderungen für das Krankenhaus negativ ausfallen. Fallpauschalen und Zusatzentgelte decken vor allem bei komplexen Behandlungsfällen wie der Lebertransplantation nicht die tatsächlichen Aufwendungen. Systemisch finanzielle Anreize zur Therapieentscheidung, die unabhängig von einer medizinischen Indikation sein können, sind vorherrschend. Schlussfolgerungen: Die Therapie von Gerinnungsstörungen stellt Ärzte, die Krankenhausverwaltungen und das G-DRG-System vor besondere Herausforderungen. Nicht selten besteht eine paradoxe Situation zwischen dem Streben nach einer bestmöglichen Patientenversorgung und einer adäquaten Leistungsvergütung des Krankenhauses. Finanzielle Anreize durch Pauschalen und Zusatzentgelte mit Mengenschwellen, die Therapieentscheidungen beeinflussen können, sind zukünftig zu eliminieren.

\author{
Keywords \\ Coagulation management · G-DRG system . \\ Point-of-care diagnostics
}

\section{Summary}

\section{Economics und Coagulation Management}

Background: A goal-directed therapy and the application of point-of-care devices has an enormous impact in the perioperative stage of congenital or chronic coagulation disorders. The cost-intensive usage of blood and coagulation products as well as the influence of patient outcome are analyzed in the G-DRG (German Diagnosis Related Groups) system and regarding the application of point-of-care devices. Method: By means of a literature review current studies are being compared and discussed. Results: The perioperative treatment of coagulation disorders is a complex matter. In addition to common laboratory diagnostics, point-of-care devices provide as well as expedite the necessary therapeutic decisions. Algorithm-based coagulation management optimizes the use of blood and coagulation products and improves patient outcome. In the G-DRG system, an optimized coagulation therapy probably influences the costs and revenue of the hospital in a negative way. DRG flat rates per case and supplementary payments do not cover the costs, especially regarding complex interventions, such as liver transplantation. Irrespective of the medical indication, systemic financial incentives for therapeutic decisions are prevalent. Conclusion: Adequate coagulation management is a major challenge for doctors, hospital administrations, and the whole G-DRG system. Most often, a paradoxical situation between the pursuit of the best possible patient care and an adequate remuneration of the hospital exists. Financial incentives by means of DRG flat rates per case and supplementary payments with volume thresholds, which can influence therapeutic decisions, should be eliminated in the future.

\section{KARGER \\ Fax +497614520714 \\ Information@Karger.com}

www.karger.com (c) 2013 S. Karger GmbH, Freiburg

1662-6664/13/0295-0312\$38.00/0

Accessible online at:

www.karger.com/vim
Thomas Ehrlich, Dipl.-Kfm.

Medizinisches System, Klinik Hirslanden

Witellikerstrasse 40, 8032 Zürich, Schweiz

thomas.ehrlich@hirslanden.ch 


\section{Einleitung}

Die chirurgische Behandlung von kritisch kranken Patienten stellt vielfältige Anforderungen an die behandelnden Ärzte. Die perioperative Phase der stationären Behandlung ist dabei von besonderer Bedeutung. Neben der eigentlichen Operation, in der es auf die chirurgischen Fähig- und Fertigkeiten der Beteiligten ankommt, sind auch andere, nicht primär chirurgische Pathologien zu behandeln. Allgemein kann konstatiert werden, dass bei Operationen immer mit teils ausgedehnten Blutverlusten zu rechnen ist. Anämie, hypovolämer Schock und eine direkte Beeinflussung der Blutgerinnung sind die Folge. Derartige Störungen nehmen Einfluss auf die Mortalität und Morbidität sowie die Krankenhausverweildauer [1]. Eine pathologische Störung der Blutgerinnung kann erworben werden (Genese einer Verletzung, operationsbedingt) oder im Vorfeld bereits bestehen (z.B. Willebrand-Jürgens-Syndrom, hereditärer Faktorenmangel). In beiden Fällen gilt es stets, in der perioperativen Phase das Gleichgewicht der Gerinnung mithilfe von Laborparametern zu überwachen und unerwünschten Störungen aktiv durch die adäquate Gabe von Blut- und Gerinnungspräparaten entgegenzuwirken.

Die Besonderheit bei der Überwachung der Blutgerinnung im Rahmen einer akuten Blutung besteht in der zeitnahen Informationsgewinnung des aktuellen Gerinnungsstatus. Die üblichen Laborparameter Prothrombinzeit (prothrombin time (PT)), aktivierte partielle Thromboplastinzeit (activated partial thromboplastin time (aPTT)), INR (international normalized ratio), Fibrinogenlevel und Thrombozytenanzahl stehen bei klassischen Ermittlungsverfahren in einem Zentrallabor mit einer durch Logistik und Laborprozesse bedingten Verzögerung von bis zu einer Stunde zur Verfügung [2]. Eine Aussage zur Thrombozytenfunktion oder einer sich entwickelnden Hyperfibrinolyse können in der Regel mit üblichen Laborparametern nicht getroffen werden [3]. Des Weiteren wird die Ermittlung der PT und aPTT im Labor in standardisierter Umgebung («in vitro») durchgeführt, sodass Rückschlüsse auf Interaktionen im Blutsystem nur eingeschränkt möglich sind und eventuell den Gerinnungsstatus verzerren [4]. Somit ist eine zeitnahe und an den Ursachen ausgerichtete Therapie in Akutsituationen mittels reiner Laboranalytik nicht möglich. Unter diesen Umständen wird bis heute in vielen Kliniken bei akuten Blutungen mit und ohne Schockzeichen des Patienten häufig eine «blinde» Therapie mit Blut- und Gerinnungspräparaten durchgeführt, die auf Erfahrungswerten und Standardverfahren beruht [5]. Unter- und Überdosierungen oder sogar die falsche oder wirkungslose Applikation von Blut- und Gerinnungspräparaten, die das Outcome des Patienten negativ beeinflussen, sind die Folge [6].

Entscheidende Fortschritte hin zu einer zielgerichteten und zeitnahen Therapie bei akuten perioperativen Störungen der Gerinnung wurden in junger Vergangenheit durch drei Faktoren mitbestimmt:
- Weitere Erkenntnisse im Verständnis der pathophysiologischen Mechanismen der Blutgerinnung.

- Die Verfügbarkeit von Point-of-Care-Verfahren zur zeitgerechteten Bestimmung von Gerinnungsparametern.

- Das detailliertere Wissen über die genaue Wirkung von applizierten Blutpräparaten und Gerinnungsfaktoren [7].

Die Point-of-Care-Geräte (beispielsweise Rotationsthrombelastometrie mittels ROTEM ${ }^{\circledR}$ (Tem International GmbH, München, Deutschland) in Kombination mit der Impedanzaggregometrie durch das Multiplate ${ }^{\circledR}$ (Verum Diagnostica $\mathrm{GmbH}$, München, Deutschland)) [8,9] werden in unmittelbarer Nähe des Patienten platziert, sodass Zeitverzögerungen durch zeitaufwendige Transporte entfallen. Damit sind erste Ergebnisse, die Rückschlüsse auf pathologische Veränderungen im Gerinnungsgleichgewicht zulassen, nach etwa 5 min schon vor Ort ersichtlich. Alternative Methoden zur Verkürzung der Analysedauer durch die Nutzung von Rohrpostanlagen zur Blutprobenübermittlung sind gemäß aktueller Studienergebnisse nur bedingt möglich [10].

Eine evidenzbasierte Interpretation der erhobenen Daten und die konsekutiv standardisierte Therapie wird mittels krankheitsspezifischer Behandlungsalgorithmen sichergestellt $[11,12]$. Einige Algorithmen berücksichtigen auch externe Einflussfaktoren wie Körpertemperatur und pH-Wert [13].

Die perioperative Nutzung dieses Behandlungsregimes unter Einbeziehung von Point-of-Care-Geräten bedingt im Vergleich zur klassischen Therapie einen zusätzlichen personellen und apparativen Aufwand. Neben Investitions- und Betriebskosten fallen zusätzliche Kosten für die Bedienung und qualitätssichernde Wartung der Geräte an. Auf der anderen Seite bewirkt eine derartige Änderung des perioperativen Gerinnungsmanagements in der Traumatologie, der Allgemeinchirurgie sowie der Herz- und Thoraxchirurgie eine signifikante Veränderung des Applikationsregimes von Blutund Gerinnungspräparaten: Während der Verbrauch von Faktorkonzentraten wie PPSB und Fibrinogen ansteigt, sinkt die Transfusionsrate von allogenen Blutprodukten wie Erythrozytenkonzentrat, Thrombozytenkonzentrat und Gefrierplasmen (fresh frozen plasma (FFP)) signifikant [14]. Durch die reduzierte Gabe von Blutprodukten und die adäquate Behandlung von nicht primär chirurgisch bedingten Gerinnungsstörungen kann das Outcome des Patienten häufig positiv beeinflusst werden. Organversagen insbesondere der Niere und Lunge durch Mikrozirkulationsstörungen nach Massentransfusionen werden verhindert, während die postoperative Beatmungs- und Intensivbehandlungsdauer reduziert wird [15]. Das Infektionsrisiko sinkt bei Patienten, wenn Transfusionen mit FFP ausbleiben [16]. Die differenzierte Gerinnungstherapie vermindert die Anzahl von postoperativen Thrombosen [17] und deren chirurgischer Behebung [18].

Bisher wurden die finanzrelevanten Auswirkungen eines geänderten Gerinnungsregimes mit dem zusätzlichen Einsatz von Point-of-Care-Geräten nur bedingt, stets kostenseitig und retrospektiv analysiert [19]. Beispielsweise wurde untersucht, 
ob die Einsparungen an Blutprodukten verrechnet mit den Mehraufwendungen für einzelne Gerinnungspräparate die Kosten, die für die zusätzlichen Point-of-Care-Untersuchungen anfallen, überschreiten [20]. Ein prospektiv randomisierter Ansatz zur Messung der möglichen finanziellen und qualitativen Effekte bei Patienten mit herz- und thoraxchirurgischen Eingriffen wurde aufgrund einer vermeintlichen Beeinflussung der Mortalität nicht zu Ende geführt. Unterstellt war eine stets positive Veränderung des Patientenoutcomes bei Algorithmus-basiertem Gerinnungsmanagement [21].

Betrachtet man nun den finanzwirksamen Aufwand und die zu erwartenden positiven Effekte, gilt es, diese nicht nur kostenseitig, sondern im Gesamtkontext des G-DRG (German Diagnosis Related Groups)-Systems mit erhaltenen Fallpauschalen und Zusatzentgelten zu bewerten.

\section{G-DRG-System und Gerinnungsmanagement}

In Deutschland regelt $\S 17 \mathrm{~b}$ Krankenhausfinanzierungsgesetz (KHG) die Einführung eines durchgängigen, leistungsorientierten und pauschalierten Entgeltsystems für Krankenhäuser im stationären Umfeld. Die Festlegung der verschiedenen Budgetrahmen (Fallpauschalen, Zusatzentgelte, ergänzende Entgelte) ist im Krankenhausentgeltgesetz (KhEntG) enthalten. Im Auftrag der Selbstverwaltungspartner im Gesundheitswesen wurde die Verantwortung für die Einführung, Weiterentwicklung und Pflege des Entgeltsystems an das Institut für das Entgeltsystem im Krankenhaus (InEK) übertragen. Die jährlich aktualisierten Fallpauschalen basieren auf einem Ist-Kostenansatz auf Vollkostenbasis. Die Abfrage der Daten erfolgt standardisiert gemäß einem Kalkulationshandbuch. Die Lieferung der Daten wird freiwillig von Krankenhäusern, die sich einer Vereinbarung zur Einhaltung des Kalkulationshandbuchs verpflichten, realisiert. Die Leistungsabrechnung erfolgt in der Regel unabhängig vom tatsächlich anfallenden Aufwand mit Pauschalbeträgen. Bei der Behandlung von Patienten, die an einer chronischen Koagulopathie leiden oder eine Blutgerinnungsstörung durch ein Trauma oder eine Operation erworben haben, ist oftmals die Gabe von kostenintensiven Blut- und Gerinnungsprodukten erforderlich. Der Therapieaufwand ist nicht vorhersehbar und birgt immer ein finanzielles Risiko für das Krankenhaus. Durch das hohe Preisgefüge der Produkte kann der pauschale Fallerlös, der für die Patientenbehandlung vorgesehen ist, die Behandlungskosten oftmals nicht decken [22]. Eine Risikoabdeckung durch Fallpauschalen sieht das System nicht vor. Deshalb gilt seit Einführung des G-DRG-Systems bei der Behandlung von Blutern (mit Blutgerinnungsfaktoren) eine vergütungsrechtliche Sonderregelung: In Ergänzung zu den Fallpauschalen können Zusatzentgelte abgerechnet werden.

Nach KHG § 17b Absatz 1 Satz 12 und 13 sind Zusatzentgeltvereinbarungen, die einen zusätzlichen finanziellen Aufwand in der Behandlung vergüten, verhandelbar. Zu unter- scheiden sind bundeseinheitliche und regional (zwischen dem Krankenhaus und den Kostenträgern) vereinbarte unbewertete Zusatzentgelte. Die Höhe der jeweiligen Zusatzentgelte wird anhand von Empfehlungen des InEK kalkuliert und beruht prinzipiell auf den Bruttomaterialkosten [23].

Die Kostenträger vereinbaren in vielen Fällen für den Verbrauch von bestimmten Produkten untere Mengenschranken pro Fall, die überschritten werden müssen, um Zusatzentgelte abrechnen zu können. Liegt der Verbrauch eines Präparats mit einer Verbrauchsmengenschranke unterhalb der Mindestmenge, sind die Aufwendungen über den Fallpauschalenerlös abgegolten. Bis Ende 2012 waren die krankenhausindividuellen Vereinbarungen über Zusatzentgelte sehr heterogen und der Katalog der bundeseinheitlichen Zusatzentgelte für Blut- und Gerinnungsprodukte relativ klein. Seit 2013 werden im Allgemeinen die Zusatzentgelte für Blut- und Gerinnungspräparate größtenteils bundeseinheitlich geregelt. Insgesamt wurden ab dem Jahr 2013155 bundeseinheitliche Zusatzentgelte vereinbart [24]. Davon entfallen für Blut- und Gerinnungspräparate:

- ZE2013-30 Prothrombinkomplex, parenteral;

- ZE2013-47 Antithrombin III, parenteral;

- ZE2013-84 Apherese-Thrombozytenkonzentrat;

- ZE2013-94 Thrombozytenkonzentrat;

- ZE2013-107 Erythrozytenkonzentrat;

- ZE2013-108 Patientenbezogenes Thrombozytenkonzentrat;

- ZE2013-97 Behandlung von Blutern mit Blutgerinnungsfaktoren;

- ZE2013-98 Gabe von Blutgerinnungsfaktoren.

Bis auf ZE2013-84 und -108 sind überall Mindestmengen notwendig, um das Zusatzentgelt abrechnen zu können. So können z.B. Erythrozytenkonzentrate erst ab einer Menge von 6 Verbrauchseinheiten zusatzentgeltlich über ZE2013-107 vergütet werden.

Neben diesem Abrechnungskatalog gelten die allgemeinen Bestimmungen des G-DRG-Fallpauschalenkatalogs inklusive mitgeltender Anlagen. In den Anlagen 4 bzw. 6 der Fallpauschalenvereinbarung (FPV) [25] werden seit 2013 im Vergleich zu den Vorjahren unter anderem Zusatzentgelte neu definiert. Die neue Definition regelt die Zusatzentgeltauslösung für Blutgerinnungspräparate bundeseinheitlich, sodass bisherige regional gültige Zusatzentgeltvereinbarungen zwischen Krankenhäusern und Kostenträgern überwiegend nicht mehr zum Tragen kommen. Es werden zwei neue Zusatzentgelte eingeführt:

- Das ZE2013-97 ist ein unbewertetes extrabudgetäres Zusatzentgelt für die Behandlung von Blutern mit Blutgerinnungsfaktoren bei angeborener oder dauerhaft erworbener Gerinnungsstörung. Hierzu zählt auch die Gerinnungsstörung, die nur durch eine Lebertransplantation heilbar wäre. Es ist zu beachten, dass das ZE2013-30 (Prothrombinkomplex, parenteral) nur dann abgerechnet werden kann, wenn ZE2013-97 (Behandlung von Blutern mit Blutgerinnungsfaktoren) nicht geltend gemacht wird. Alle Gaben von vorgesehenen Präparaten werden auf Einzelkostenbasis abgerechnet. 


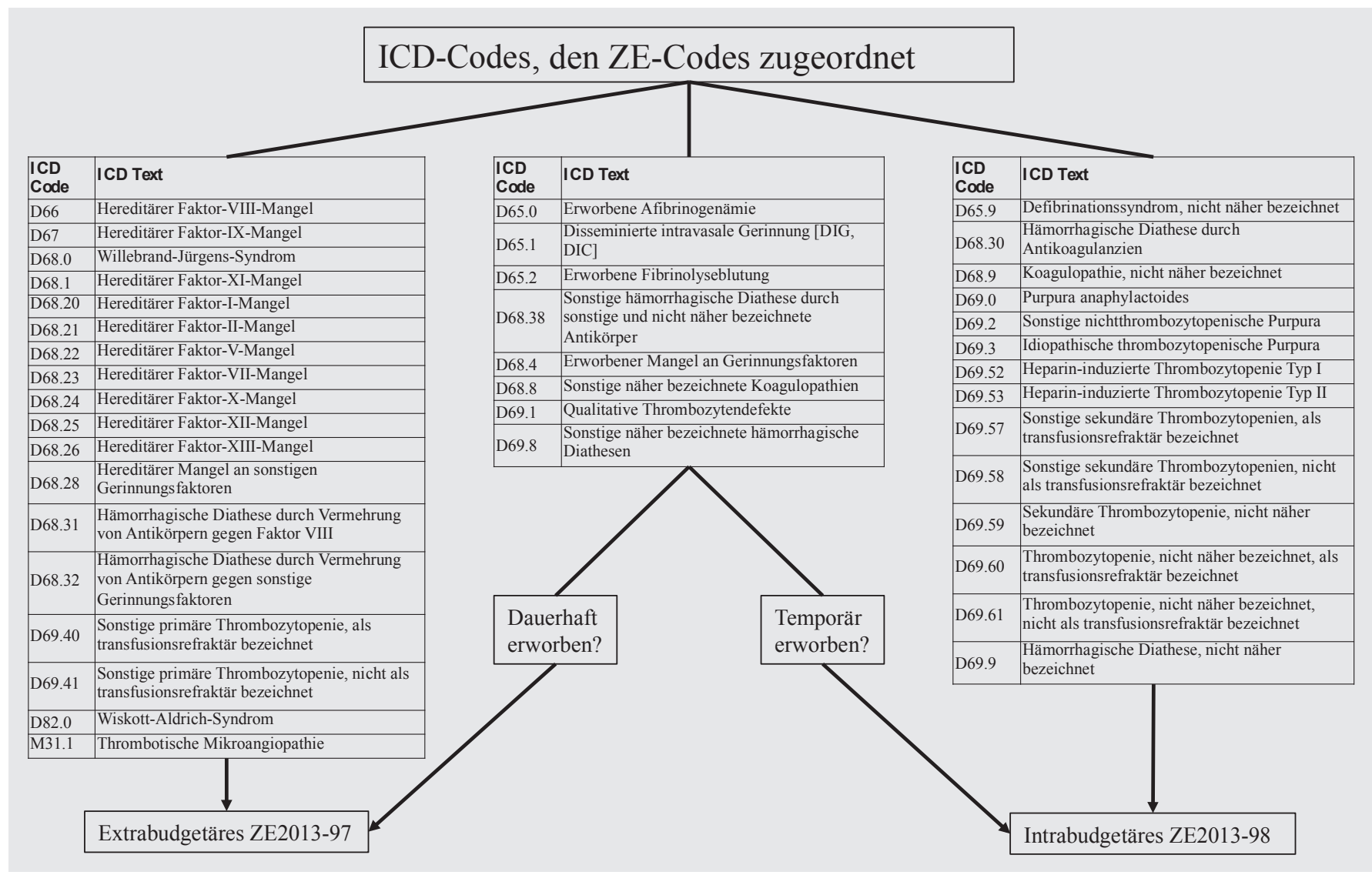

Abb. 1. ICD(International Classification of Diseases)-Codes, die beim Fall kodiert werden müssen, um die unterschiedlichen Zusatzentgelte (ZE) auszulösen (vergleiche G-DRG-Fallpauschalenkatalog Anlage 7, 2013).

- Das ZE2013-98 (Gabe von Blutgerinnungsfaktoren) ist ebenfalls ein unbewertetes, jedoch intrabudgetäres $\mathrm{Zu}$ satzentgelt, das bei erworbener Koagulopathie ausgelöst werden kann. Für das ZE2013-98 gilt eine Kostenschwelle bei 15000 EUR. Liegen die Ist-Kosten für relevante Präparate unterhalb dieser Schranke, ist eine Geltendmachung über dieses Zusatzentgelt nicht möglich. Überschreiten die Ist-Kosten die Schranke, können alle anfälligen Kosten geltend gemacht und zusätzlich zur Fallpauschale abgerechnet werden.

Diese Zusatzentgelte werden über die Kodierung bestimmter Diagnosen (Abb. 1) und Prozeduren (Abb. 2) angesteuert. Einige Diagnosen wie beispielsweise D65.2 (Erworbene Fibrinolyseblutung) werden bei dauerhaftem Erwerb der Störung dem ZE2013-97 oder bei temporärer Störung dem ZE2013-98 zugeordnet.

\section{Das Gerinnungsmanagement im G-DRG-System}

Wegen der oftmals notwendigen Anwendung von hochpreisigen Präparaten bei der Therapie von Blutgerinnungsstörungen ist die Abbildung in Fallpauschalen nicht ausreichend, sodass meistens eine zusätzliche Aufwandsentschädigung über die Abrechnung von Zusatzentgelten notwendig ist [26]. Die vereinbarten Mengenschranken bei einigen Blut- und Gerinnungspräparaten, die überschritten werden müssen, um Zusatzentgelte abrechnen zu können, implizieren allerdings, dass der finanzielle Aufwand bei geringen Mengen über die Fallpauschalen abgedeckt wird.

Aus den vorliegenden gesetzlichen Vorgaben und Anreizen ergeben sich für den behandelnden Arzt bzw. für die Krankenhäuser Konsequenzen für die Behandlung von Patienten, die eine akute Blutung erleiden. Der perioperative Einsatz von Blut- und Gerinnungspräparaten sollte stets gezielt und nur im Rahmen eines standardisierten Behandlungsregimes erfolgen, um die Verabreichung von Blut- und Gerinnungsprodukten im Vergleich zu einer «blinden» Therapieform signifikant zu verbessern und die Komplikationen durch einen überschießenden Einsatz von Blutprodukten zu minimieren. Eine Algorithmusbasierte Therapie, die spezielle Krankheitsspezifika einbezieht, die Nutzung von Point-of-Care-Methoden zum Monitoring der Gerinnungsparameter sowie die Prüfung von Einsatz und Wirkung applizierter Präparate sollten den Standard in der perioperativen Gerinnungstherapie darstellen. In die Entscheidungsfindung zur gezielten Therapie fließen immer auch die klinischen Parameter des Patienten wie Schockzeichen, aktive Blutungen, Hypothermie, Azidose und vieles mehr ein. 


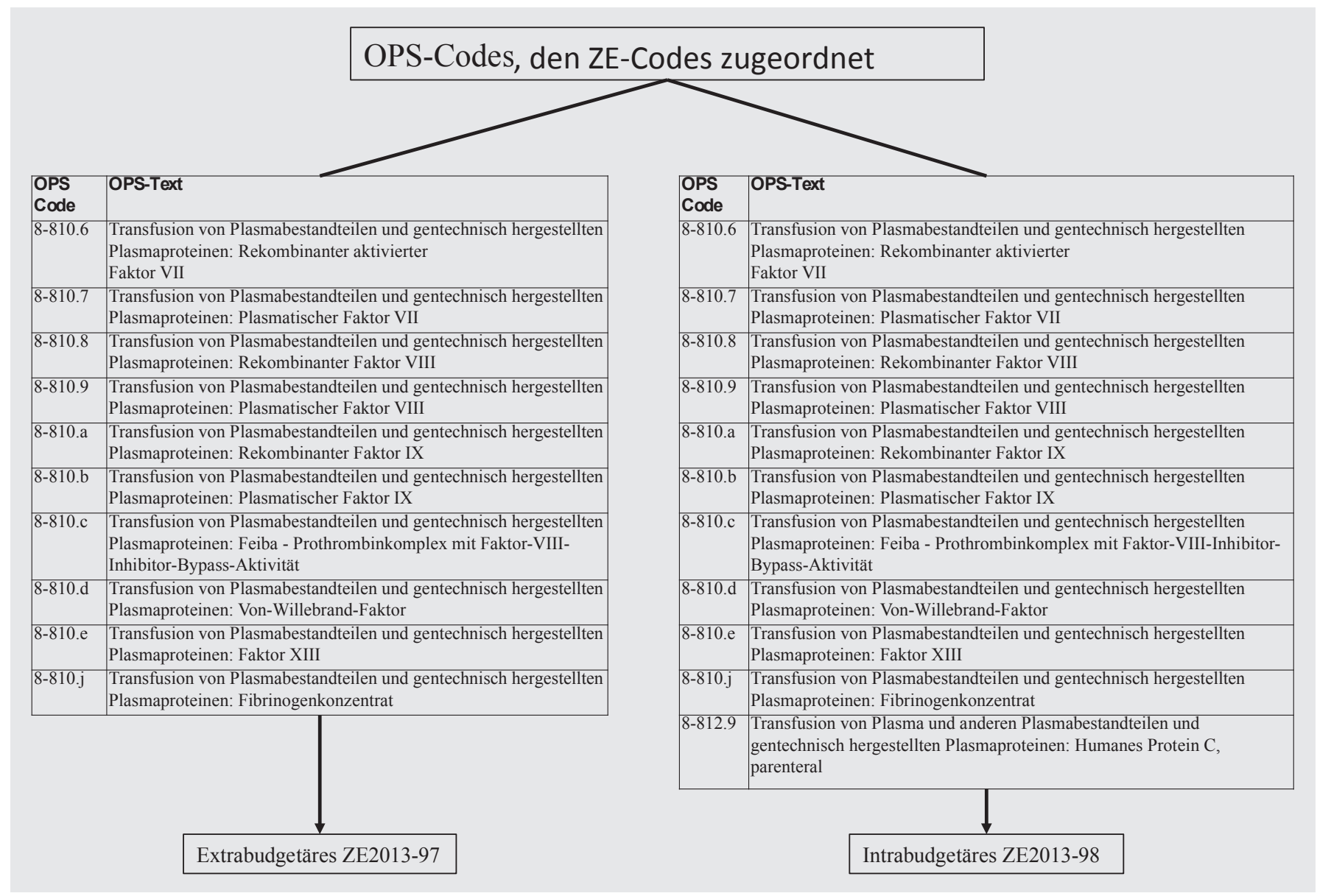

Abb. 2. OPS(Operationen- und Prozedurenschlüssel)-Codes, die beim Fall kodiert werden müssen, um die unterschiedlichen Zusatzentgelte auszulösen (vergleiche G-DRG-Fallpauschalenkatalog Anlage 6, 2013).

Verschiedene Analysen haben gezeigt, dass im Vergleich zur ungesteuerten Therapie ohne Algorithmus und Pointof-Care-Geräte der Einsatz von Erythrozytenkonzentraten, Thrombozytenkonzentraten und FFP deutlich verringert werden kann [27]. Unter diesen Regimen sind bei optimalen Voraussetzungen sogar Lebertransplantationen ohne Blutersatz möglich [28]. Die Algorithmus-basierte Therapie führt also zu einer Verbesserung der Patientenbehandlungsqualität und der Patientensicherheit. Konterkariert wird dieser moderne Ansatz durch die derzeitige Finanzierungssituation im G-DRGSystem: Werden allogene Blutprodukte verwendet und liegen Mengenschwellen für diese zur Auslösung von Zusatzentgelten vor, besteht aus Sicht des Krankenhauses der finanzielle Anreiz, die Mengenschranke zu überschreiten, um anfällige Kosten mithilfe des Fallerlöses und den Zahlungen für $\mathrm{Zu}$ satzentgelte mindestens zu decken. Sind keine Mengenschranken zur Auslösung von Zusatzentgelten vorhanden, erfolgt die Gabe von entsprechenden Präparaten mehr oder minder unabhängig von finanziellen Anreizen und nach Indikation.

\section{Beispiel: Erythrozytentransfusion bei akuten Blutungen}

Dies soll an einem einfachen Beispiel illustriert werden: Die Gabe von 5 Erythrozytenkonzentraten im Rahmen der
Behandlung einer erworbenen Anämie bzw. Koagulopathie lässt aufgrund der Mindestmengenschranke bei 6 Einheiten keine Geltendmachung eines Zusatzentgeltes zu. Der finanzielle Aufwand, der durch die Gabe des Blutes anfällt, ist durch die pauschalen Fallerlöse abzudecken, was eine potenzielle Gewinnspanne in einer Kostenträgerbetrachtung minimiert oder gar eliminiert. Werden jedoch 6 Einheiten verabreicht, werden sämtliche Kosten durch die Geltendmachung des $\mathrm{Zu}$ satzentgelts ZE2013-107 gedeckt, und die Gewinnspanne erhöht sich deutlich. Somit wird bei einem Präparat mit einer Mindestmengenschranke ein finanzieller Anreiz geschaffen, aus Kostendeckungsgründen die Mindestmenge - ungeachtet einer medizinischen Indikation - zu verabreichen. Dies gilt zumindest bei Präparaten, bei denen Nutzen und Risiko einer zusätzlichen Einheit relativ klein erscheinen (Grenznutzentheorie). Zwar widerspricht ein derartiges Behandlungsregime den Grundlagen einer nutzen- und risikobezogenen Therapie, allerdings wird nicht selten der finanzielle Nutzen für das Krankenhaus höher bewertet. Diese Anreizproblematik besteht im gesamten DRG-System an den Stellen, wo quantifizierte Mengengrenzen eine finanzielle Auswirkung auf die Erlösseite des Krankenhauses haben. Dieses Beispiel zeigt deutlich das medizinethische Problem auf, dass eine 
Kostendeckung in bestimmten Fällen nur mit einem erhöhten Patientenrisiko erzielt werden kann. Die Mengen- und Kostenschranken, die überschritten werden müssen, um finanzrelevante Effekte durch die Geltendmachung von Zusatzentgelten auszulösen, schaffen somit Anreize, die vom Gesetzgeber in dieser Form nicht beabsichtigt sein können. Das Prinzip des abnehmenden Grenznutzens bei der Applikation einer zusätzlichen Einheit von Blutprodukten wie FFP oder Thrombozytenkonzentrate in Bezug auf das wachsende Genesungsrisiko (beispielsweise für Infektionen oder eine Verlängerung der postoperativen Beatmungsdauer) gilt explizit nicht.

\section{Beispiel: Lebertransplantation}

Weitere finanzielle Nachteile werden im Folgenden am Beispiel der Lebertransplantation und deren pauschalen DRG-Vergütung illustriert. Die Lebertransplantation im deutschen DRG-System kann mit drei DRGs abgebildet werden:

- A01A: Lebertransplantation mit Beatmung >179 h;

- A01B: Lebertransplantation mit Beatmung $>59$ und $<180 \mathrm{~h}$ oder mit Transplantationsabstoßung oder mit kombinierter Dialyse/Leberersatztherapie;

- A01C: Lebertransplantation ohne Beatmung >59 h.

Dabei reichen die Pauschalen von 31 232,80 EUR (A01C) über 40 196,40 EUR (A01B) bis 92 502,10 EUR (A01A) [29]. Bei allen Pauschalen liegen die zugeordneten Personalkosten bei über $50 \%$ der Gesamtkosten und die Kosten für medizinische und nicht medizinische Infrastruktur bei ca. 20\%. Somit ist ein Fixkostenanteil mit über $70 \%$ festzustellen, der stets bei einer Lebertransplantation anfällt. Die tatsächlichen Kosten können jedoch stark schwanken und überschreiten oft und insbesondere bei schwierigen und aufwendigen Therapien die pauschale Vergütung [30]. Wie man aus den Klassifikationen der einzelnen Fallpauschalen ersehen kann, ist die Eingruppierung und Kodierung stark von der Beatmungsdauer abhängig. Die Beatmungsdauer wiederum ist bei einer Lebertransplantation unter anderem abhängig von einer starken Blutung in der perioperativen Phase [31]. Die frühzeitige Extubation nach einer Lebertransplantation mit der Anwendung von Fast-Track-Protokollen bei Patienten, deren klinischer Zustand einen Einschluss in den Fast-Track erlaubt, beeinflusst wiederum die Beatmungsdauer und somit die DRG-Kodierung [32]. Zusätzlich gilt die intraoperative Transfusion von Erythrozytenkonzentraten [33] und Thrombozytenkonzentraten [34] jeweils als unabhängiger Risikofaktor für das Outcome und somit das Überleben des Patienten. Die genannten medizinischen Zusammenhänge sprechen eindeutig für ein optimiertes Gerinnungsmanagement in der perioperativen Phase der Lebertransplantation, in der Blutund Gerinnungspräparate gezielt und kontrolliert eingesetzt werden.

Im Widerspruch dazu steht die Refinanzierung der Therapieaufwendungen: Verbessert sich das Outcome des Patienten insbesondere durch Verkürzung der DRG-relevanten Beatmungs- dauer [35], ferner durch die frühzeitige Entlassung von der Intensivstation bzw. sogar aus dem Krankenhaus, kann dies auf der anderen Seite zur Folge haben, dass sich die Erlössituation für das Krankenhaus verschlechtert: Durch die eventuell reduzierte Beatmungsdauer bei einer Lebertransplantation ist zu erwarten, dass die Kodierung des Falls im Vergleich mit einem Patienten, der wie bisher üblich länger beatmet werden musste, eine niedrigere Fallpauschale generiert. Auch verschiebt sich die Geltendmachung von diversen Zusatzentgelten, insbesondere bei der Unterschreitung von Mengenschranken, die ohne optimiertes Gerinnungsmanagement stets überschritten wurden. Derartige Verschiebungen können finanzielle Nachteile mit sich bringen. Das seit 2013 geltende Zusatzentgelt mit der Kostenschwelle bei 15000 EUR kann bei einer Lebertransplantation durch den optimierten Einsatz von Blut- und Gerinnungsfaktoren eventuell nicht überschritten werden, sodass derartige Ausgaben über die Fallpauschale abgedeckt werden müssen. Eine frühere Entlassung des Patienten führt bei der Betrachtung der Kostenträgerebene und den oben dargestellten Fixkostenanteilen der Patientenbehandlung durch Vorhaltung von Infrastruktur und Personal zu keinem direkten Einspareffekt. Diese negativen Erlöseffekte lassen sich aktuell nur ausgleichen, indem durch die schnellere Entlassung von der Intensivstation und aus dem Krankenhaus konsequent ein weiterer Patient behandelt wird und der zusätzlich mögliche Fallerlös einen positiven finanzwirksamen Effekt erzielen kann.

Solche Problemsituationen bei der Kodierung von Fällen und deren pauschalen Leistungsvergütung behindern förmlich das Streben nach einer verbesserten Patientenbehandlung wie im Rahmen eines optimierten Gerinnungsmanagements. Zwar stellen derartige Programme einen eindeutigen Mehrwert für die Patienten dar, jedoch ist der finanzielle Anreiz zur Anschaffung von zusätzlichen Gerätschaften und deren konsequenter Anwendung im Krankenhaus wegen der systembedingten Gefahr eines Erlösrückgangs und relativ konstant anfallenden Kosten durch nicht beeinflussbare Fixkostenanteile nicht gegeben. Ähnlich wie bei der Geltendmachung von $\mathrm{Zu}$ satzentgelten sind Schwellenwerte, deren Überschreitung die Fallvergütung steuern, kritisch zu betrachten. Im Fall der Lebertransplantation wird der Fallerlös überwiegend über die Beatmungsdauer festgelegt. Eine verkürzte Beatmungsdauer durch ein modernes Behandlungsregime wirkt sich somit eher negativ auf die Erlössituation aus. Somit besteht für die Krankenhausleitung ein finanzieller Anreiz, die Schwellenwerte zur Fallerlösverbesserung auch in medizinisch unbegründeten Fällen stets zu überschreiten - eine paradoxe Situation zwischen dem Streben nach einer bestmöglichen Patientenversorgung und einer adäquaten Leistungsvergütung des Krankenhauses.

\section{Notwendige Anpassungen im G-DRG-System}

Um diese Schwachstellen in der Krankenhausrefinanzierung zukünftig auszugleichen, muss seitens InEK oder der 
Kostenträger erwogen werden, ein auf Algorithmen und Point-of-Care-Geräte gestütztes Gerinnungsmanagement zu fordern, um gewisse DRG oder Zusatzentgelte kodieren und abrechnen zu können. Aufgrund der nachweislich positiven Effekte bei einem gezielten Einsatz von Transfusionen und Gerinnungspräparaten sollte ein medizinischer Anreiz mit Kostendeckung geschaffen werden. Im Rahmen einer Lebertransplantation ist eine differenzierte Gruppierung der komplexen Behandlung mit Einschluss der Genese der Erkrankung, des MELD(Model for End-Stage Liver Disease)-Scores und weiterer klinisch relevanter Prozessvariablen zur aufwandsgerechten Leistungsvergütung zwingend erforderlich.

\section{Fazit}

Das perioperative Gerinnungsmanagement und dessen Optimierung stellt sowohl für die behandelnden Ärzte als auch für die Administration der Krankenhäuser eine besondere Herausforderung dar. Aktuelle Forschungen zeigen, dass die Algorithmus-basierte Gerinnungstherapie in Verbindung mit Point-of-Care-Geräten den gezielten Einsatz von allogenen Blutprodukten und Gerinnungspräparaten optimiert. Die positive Beeinflussung des Patientenoutcomes ist die unmittelbare Folge.

Im G-DRG-System bildet sich diese Behandlung nur unzureichend $a b$. Weiterhin besteht die paradoxe Situation, dass eine verbesserte Behandlungsqualität zu Erlöseinbußen führen kann. Etwaige Mehrkosten werden oftmals bei Überschreitung von Schwellenwerten über Zusatzentgelte gedeckt. Werden Schwellenwerte nicht überschritten, können die Pauschalvergütung und Zusatzentgelte derart beeinflusst werden, dass finanzielle Nachteile für das Krankenhaus entstehen und kontroverse Anreize verursacht werden, die vom Gesetzgeber nicht gewollt sein können.

Im Rahmen der kontinuierlichen Optimierung der DRGVergütungssystematik sind die diskutierten Schwachstellen auszugleichen. Die Neuordnung der Zusatzentgelte bei der Behandlung von Blutern sowie der Schwellenschranke zu deren Auslösung ist aus Sicht des Autors erneut reformbedürftig. Die argumentativ hergeleiteten Situationen und Aussagen sind in zukünftigen Studien beispielsweise mit retrospektiven Pair-Match-Verfahren oder Kohortenanalysen zu validieren.

\section{Disclosure Statement}

Thomas Ehrlich ist Management-Assistent im Medizinischen System der Klinik Hirslanden in Zürich. Er verfolgt als externer Doktorand am Lehrstuhl für Betriebswirtschaftslehre, insbesondere Management des Gesundheitswesens an der Universität des Saarlandes in Saarbrücken, Deutschland, den Forschungsansatz gesundheitsökonomischer Effekte im G-DRG-Umfeld der Einführung einer optimierten Blutgerinnungsdiagnostik und -therapie.

\section{Literatur}

1 Musallam KM, Tamim HM, Richards T, Spahn DR, Rosendaal FR, Habbal A, Khreiss M, Dahdaleh FS, Khavandi K, Sfeir PM, Soweid A, Hoballah JJ, Taher AT, Jamali FR: Preoperative anaemia and postoperative outcomes in non-cardiac surgery: a retrospective cohort study. Lancet 2011 378:1396-1407.

2 Toulon P, Ozier Y, Ankri A, Fleron MH, Leroux G, Samama CM: Point-of-care versus central laboratory coagulation testing during haemorrhagic surgery. A multicenter study. Thromb Haemost 2009:101:394-401.

$\checkmark 3$ Rahe-Meyer N, Winterhalter M, Boden A, Froemke C, Piepenbrock S, Calatzis A, Solomon C: Platelet concentrates transfusion in cardiac surgery and platelet function assessment by multiple electrode aggregometry. Acta Anaesthesiol Scand 2009;53: $168-175$.

4 Martini WZ, Cortez DS, Dubick MA, Park MS, Holcomb JB: Thrombelastography is better than PT, aPTT, and activated clotting time in detecting clinically relevant clotting abnormalities after hypothermia, hemorrhagic shock and resuscitation in pigs. J Trauma 2008;65:535-543.

$\checkmark 5$ Avidan MS, Alcock EL, Da Fonseca J, Ponte J, Desai JB, Despotis GJ, Hunt BJ: Comparison of structured use of routine laboratory tests or nearpatient assessment with clinical judgement in the management of bleeding after cardiac surgery. Br J Anaesth 2004:92:178-186.
6 Ganter MT, Spahn DR: Active, personalized, and balanced coagulation management saves lives in patients with massive bleeding. Anesthesiology 2010;113:1016-1018.

7 Tanaka KA, Kor DJ: Emerging haemostatic agents and patient blood management. Best Pract Res Clin Anaesthesiol 2013;27:141-160.

8 Glas M, Mauer D, Brün K, Volk T, Kreuer S: Einführung in die POC-Methodik - Indikationen und Limitationen. Anasthesiol Intensivmed Notfallmed Schmerzther 2013;48:324-332.

$\checkmark 9$ Pape A, Weber CF, Stein P, Zacharowski K: ROTEM $^{\circledR}$ and Multiplate ${ }^{\circledR}$ - a suitable tool for POC? ISBT Science Series 2010;5:161-168.

10 Glas M, Mauer D, Kassas H, Volk T, Kreuer S: Sample transport by pneumatic tube system alters results of multiple electrode aggregometry but not rotational thromboelastometry. Platelets 2013;24: 454-461.

11 Görlinger K, Jambor C, Hanke A, Dirkmann D, Adamzik M, Hartmann M, Rahe-Meyer N: Perioperative coagulation management and control of platelet transfusion by point-of-care platelet function analysis. Transfus Med Hemother 2007;34: 396-411.

12 Meybohm P, Zacharowski K, Weber CF: Point-ofcare coagulation management in intensive care medicine. Crit Care 2013;17:218

13 Weber CF: Modern coagulation management reduces the transfusion rate of allogenic blood products. Anasthesiol Intensivmed Notfallmed Schmerzther 2012;47:418-424.
14 Ehrlich T, Glas M, Mauer D, Volk T, Kreuer S: Introducing a Thrombelastometry and Impedance Aggrometry Based Point-of-Care Coagulation Management - Results of a One Year Pilot Test. Annual Meeting of the American Society of Anesthesiologists 2012;A684.

15 Nienaber U, Innerhofer P, Westermann I, Schochl H, Attal R, Breitkopf R, Maegele M: The impact of fresh frozen plasma vs coagulation factor concentrates on morbidity and mortality in traumaassociated haemorrhage and massive transfusion. Injury 2011;42:697-701.

16 Sarani B, Dunkman WJ, Dean L, Sonnad S, Rohrbach JI, Gracias VH: Transfusion of fresh frozen plasma in critically ill surgical patients is associated with an increased risk of infection. Crit Care Med 2008;36:1114-1118.

17 Görlinger K, Dirkmann D, Hanke AA: Potential value of transfusion protocols in cardiac surgery. Curr Opin Anaesthesiol 2013;26:230-243.

18 Görlinger K, Dirkmann D, Hanke AA, Kamler M, Kottenberg E, Thielmann M, Jakob H, Peters J: First-line therapy with coagulation factor concentrates combined with point-of-care coagulation testing is associated with decreased allogeneic blood transfusion in cardiovascular surgery: a retrospective, single-center cohort study. Anesthesiology 2011;115:1179-1191.

19 Heindl B: Perioperative coagulation monitoring medical and economic aspects. Anasthesiol Intensivmed Notfallmed Schmerzther 2010;45:292-296. 
20 Spalding GJ, Hartrumpf M, Sierig T, Oesberg N, Kirschke CG, Albes JM: Cost reduction of perioperative coagulation management in cardiac surgery: value of 'bedside' thrombelastography $\left(\right.$ ROTEM $\left.^{\circledR}\right)$. Eur J Cardiothorac Surg 2007;31: 1052-1057.

21 Weber CF, Görlinger K, Meininger D, Herrmann E, Bingold T, Moritz A, Cohn LH, Zacharowski K: Point-of-care testing: a prospective, randomized clinical trial of efficacy in coagulopathic cardiac surgery patients. Anesthesiology 2012;117:531-547.

22 Bauer M, Ostermann H: DRGs in Transfusion Medicine and Hemotherapy in Germany. Transfus Med Hemother 2012;39:60-66.

23 InEK Kalkulationshandbuch 2013. www.g-drg.de/ cms/Kalkulation2/DRG-Fallpauschalen_17b_ KHG/Kalkulationshandbuch.

24 GKV: Kommentar zum G-DRG System 2013. www.gkv-spitzenverband.de/krankenversicherung/ krankenhaeuser/drg_system/g_drg_2013/drg_system_1.jsp.

25 InEK Vereinbarung zum Fallpauschalensystem für Krankenhäuser für das Jahr 2013. www.g-drg.de/ cms/G-DRG-System_2013/Abrechnungsbestimmungen/FPV_2013.
26 Heimig F: Das G-DRG-System 2013 - Klassifikation und Bewertung. 35. Deutscher Krankenhaustag 2012. www.deutscher-krankenhaustag.de/de/ vortraege/pdf/Heimig_endgueltig_2.pdf.

27 Weber CF, Zacharowski K: Perioperative Point-ofCare-Gerinnungsdiagnostik. Dtsch Ärztebl 2012; 109:369-375.

28 Mauer D, Ehrlich T: Kostenpunkt Blutprodukte. Südwestdeutsche Anästhesietage 2011.

29 InEK Report-Browser 2011/2013. www.g-drg.de/ cms/G-DRG-System_2013/Abschlussbericht_zur_ Weiterentwicklung_des_G-DRG-Systems_und_ Report_Browser/Report-Browser_2011_2013.

30 Kraus TW, Mieth M, Schneider T, Farrenkopf I, Mehrabi A, Schemmer P, Encke J, Sauer P, Buchler MW: Cost distribution of orthotopic liver transplantation: single-center analysis under DRG-based reimbursement. Transplantation 2005;80:S97-S100.

31 Glanemann M, Langrehr J, Kaisers U, Schenk R, Muller A, Stange B, Neumann U, Bechstein WO, Falke K, Neuhaus P: Postoperative tracheal extubation after orthotopic liver transplantation. Acta Anaesthesiol Scand 2001;45:333-339.
32 Glanemann M, Busch T, Neuhaus P, Kaisers U: Fast tracking in liver transplantation. Immediate postoperative tracheal extubation: feasibility and clinical impact. Swiss Med Wkly 2007;137:187-191.

33 Cacciarelli TV, Keeffe EB, Moore DH, Burns W, Busque S, Concepcion W, So SK, Esquivel CO: Effect of intraoperative blood transfusion on patient outcome in hepatic transplantation. Arch Surg 1999;134:25-29.

34 de Boer MT, Christensen MC, Asmussen M, van der Hilst CS, Hendriks HG, Slooff MJ, Porte RJ The impact of intraoperative transfusion of platelets and red blood cells on survival after liver transplantation. Anesth Analg 2008;106:32-44.

35 Ehrlich T: Gesundheitsökonomische Untersuchung der Einführung eines point-of-care Blutgerinnungsmanagements an einem deutschen Universitätsklinikum. Annual Meeting of GOR Health Care Management 2012.www.gor-hcm.de/htdocs/ abstracts/2012/HCM_Abstract_Ehrlich_2012.pdf. 